\title{
Predictors of intractable epilepsy in childhood: A case control study
}

\author{
P Sangeetha $^{1}$, K Anoop ${ }^{2 *}$ \\ ${ }^{1}$ Assistant Professor, ${ }^{2}$ Consultant Paediatrician, ${ }^{1}$ Dept. of Pediatrics, ${ }^{1}$ Govt. Medical College, Palakkad, Kerala, ${ }^{2}$ Al Madeena \\ Institute of Medical Sciences (ALMAS) Kottakkal, Malappuram, Kerala, India
}

*Corresponding Author: K Anoop

Email: dranoop_paeds2007@yahoo.co.in

\begin{abstract}
Aims: To find out the predictors of intractable epilepsy in childhood and to identify these children prone for intractability at the earliest.

Settings and Design: It is a case control study done at Department of Pediatric Neurology at a tertiary care hospital.

Methods and Materials: 50 cases and 50 controls were enrolled in the study. Cases were defined as 'epileptic patients who have seizure frequency of at least 1 attack per month for at least six months despite receiving two antiepileptic drugs with adequate dosage and compliance'. Detailed history, neurologic examination, EEG and neuroimaging were done in all patients to find out factors predicting intractability.

Results: A total of 50 patients with intractable epilepsy and 50 patients with well controlled seizures were studied. A female preponderance was seen among case group, females constituting 54\% and males $46 \%$. Significant association with a $\mathrm{p}$ value $<0.001$ was found between seizure onset less than 1 year and intractable epilepsy. Presence of myoclonic seizure in the case group was found to be statistically significant with a p-value 0.006 . History of birth asphyxia, neonatal seizures, developmental delay, status epilepticus and presence of microcephaly were also significant risk factors for the development of intractable epilepsy. Common MRI findings were cerebral atrophy, neuronal migration disorders and corpus callosal agenesis.
\end{abstract}

Conclusions: Early identification of these children prone to develop intractable seizures is critical for parental counselling, selecting patients for more intensive investigations and treatment, such as early consideration for epilepsy surgery.

Keywords: Intractable epilepsy, Myoclonic seizures, Neonatal seizures.

\section{Introduction}

Epilepsy is a common neurological disorder affecting 3-6/1000 children. Approximately $70-80 \%$ will be controlled with single drug and $10-15 \%$ require combination chemotherapy. However $10-20 \%$ of epileptic patients are resistant to therapy, the condition, so called 'intractable epilepsy'., ${ }^{1,3}$ While a great deal is known about seizures and epilepsy, little is known about the identification and causes of intractable epilepsy. ${ }^{14}$ Such intractable epilepsy impose considerable socioeconomic and psychological constraint on the individual patient and cast a substantial burden on health and welfare resources. Early identification of these children, prone to develop intractable seizures is critical for parental counselling, selecting patients for more intensive investigations and treatment, such as early consideration for epilepsy surgery.

Though intractable epilepsy has been extensively studied in developed countries, there are not many studies done in developing countries. In India, there are some studies done in North India, but not in South India. The present study has been done in a tertiary care hospital in South India to find out children prone for intractability at the earliest. Aim of the study is to find out the predictors of intractable epilepsy in childhood and to identify these children prone for intractability at the earliest.

\section{Subjects and Methods}

It was a case control study conducted in Department of Pediatric Neurology at a tertiary care hospital in South India. Epileptic children in the age group six months to 12 years were included in the study. A total of 50 cases and 50 controls were included in the study. Cases were defined as 'Epileptic patients who have seizure frequency of at least one attack per month for at least six months despite receiving two antiepileptic drugs with adequate dosage and compliance'. Controls were defined as 'children with epilepsy who have adequate control of seizures for more than six months with one or two drugs'.

Detailed history and neurologic examination were done in all patients and entered in a pre-structured proforma. Seizures were classified using International League Against Epilepsy (ILAE) classification. Factors analyzed included the following: details of pharmacotherapy like number of drugs, duration after starting two drugs, dosage and compliance, age at onset of seizures, type of seizures, frequency of 
seizures, history of birth asphyxia, neonatal seizures, developmental delay and history of status epilepticus. Detailed examination was done to look for microcephaly, focal neurologic deficits, neurocutaneous markers etc.. Electroencephalogram and neuroimaging was performed in all patients to determine etiology and classification of epilepsy. Cranial computed tomography was done in all patients. MRI scan was done in most of the patients except a few where CT scan was diagnostic and a small number of patients who could not afford MRI.

Association between factors analyzed and outcome was determined using chi-square test. Odds ratio and $95 \%$ confidence interval was calculated using univariate analysis. Multivariate analysis by logistic regression method was done to rule out confounding factors and to find out independent predictors of outcome.

\section{Results}

A total of 50 patients with intractable epilepsy and 50 patients with well controlled seizures were studied. A female preponderance was seen among case group, females constituting 54\% and males $46 \%$. The difference was statistically significant with $p$ value 0.026 and odds ratio of 2.495. Majority of patients in case group $32(64 \%)$ had seizure onset <one year of age as against 11 patients (22\%) in control group. This was found to be statistically significant with a $\mathrm{p}$ value of 0.000 , Odds ratio of 6.3 and $95 \%$ Confidence Interval of $2.604-15.25$.

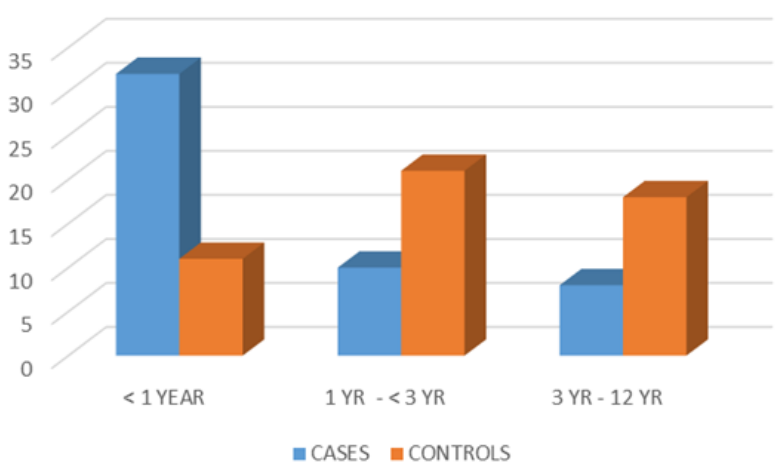

Fig. 1: Age at onset of seizures

In this study majority of the patients with intractable epilepsy were taking three drugs. Maximum number of antiepileptic drugs taken was six drugs, being taken by two patients. Most common seizure type among cases as well as controls was generalized tonic clonic seizures (64\% in case group and 56\% in control group). Seven patients (14\%) in case group had myoclonic seizures, whereas none among control group had myoclonic seizures. This difference was found to be statistically significant with a $p$ value of 0.006. Mixed seizure type was also found to be more common in case group. Most of the cases had daily seizures, before starting treatment when compared to eight in the control group and this difference was found to be statistically significant with a $p$ value of 0.000 , Odds ratio of 11.15 and $95 \%$ CI of 4.26-29.18.

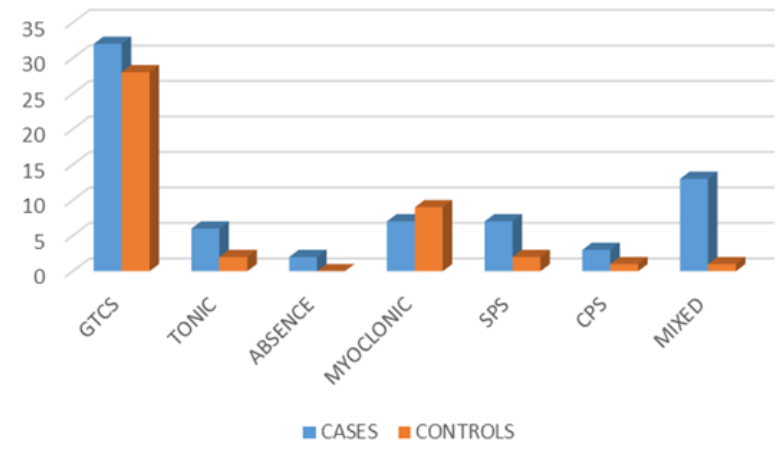

Fig. 2: Type of seizures

34 patients $(68 \%)$ in case group had developmental delay against 12 patients $(24 \%)$ in control group ( $\mathrm{p}$ value -0.000$)$. $60 \%$ of case had history of status epilepticus whereas only six \% of controls had status epilepticus $(p$ value $=0.000) .18$ patients with intractable epilepsy had history of birth asphyxia whereas only four patients $(8 \%)$ with well controlled epilepsy had birth asphyxia ( $\mathrm{p}$ value $=0.001$ ). History of neonatal seizures was found in $15(30 \%)$ cases as against $4(8 \%)$ controls ( $p$ value $=0.005)$. History of febrile seizure was found in $12 \%$ cases and $18 \%$ of controls.

Among cases, seven patients had hemiplegia, 13 patients had quadriplegia, 17 patients had microcephaly and in 23 patients there were no deficit. Among these presence of microcephaly was found to be statistically significant. Any abnormality in neurological examination was found to be statistically significant with a $\mathrm{p}$ value 0.000 . CT scan was normal in most of the cases. The most common CT abnormality was cerebral atrophy seen in seven cases $(14 \%)$ followed by gliosis in five cases $(10 \%)$. Most common MRI findings were cerebral atrophy $9(18 \%)$ in cases and ring enhancing lesion $8(16 \%)$ in controls. The other common MRI findings among case group were neuronal migration disorders $6(12 \%)$, corpus callosal agenesis $4(8 \%)$ and gliosis $5(10 \%)$. In this 
study neither CT nor MRI abnormality was found to be statistically significant with intractable epilepsy in the study population.

The most common EEG abnormality was subcortical seizure activity $15(30 \%)$ followed by background abnormality $12(24 \%)$. EEG was normal in $17(34 \%)$ cases and 33(66\%) controls. EEG abnormality was found to be statistically significant with a $\mathrm{p}$ value of 0.001
Multivariate analysis was done by multiple logistic regression method to examine association between intractable epilepsy and potential predictive factors. Female sex, multiple seizures before starting treatment, neurologic impairment, microcephaly and EEG abnormalities were found to be independent predictors of intractable seizures.

Table 1: Factors predicting Intractable Epilepsy

\begin{tabular}{|c|c|c|c|c|c|c|c|}
\hline \multirow{2}{*}{ Parameter } & \multicolumn{2}{|c|}{ Case } & \multicolumn{2}{c|}{ Control } & \multirow{2}{*}{ P Value } & OR & \multirow{2}{*}{ 95\% CI } \\
\cline { 2 - 5 } & $\mathrm{n}$ & $\%$ & $\mathrm{n}$ & $\%$ & & & \\
\hline Developmental Delay & 34 & $68 \%$ & 12 & $24 \%$ & 0.000 & 6.72 & $2.79-16.22$ \\
\hline H/O Status Epilepticus & 30 & $60 \%$ & 3 & $6 \%$ & 0.000 & 23.5 & $6.42-85.9$ \\
\hline Birth Asphyxia & 18 & $36 \%$ & 4 & $8 \%$ & 0.001 & 6.46 & $2.00-20.91$ \\
\hline Neonatal seizures & 15 & $30 \%$ & 4 & $8 \%$ & 0.005 & 4.92 & $1.50-16.15$ \\
\hline Seizure onset <1 year & 32 & $64 \%$ & 11 & $22 \%$ & 0.000 & 6.3 & $2.604-15.25$ \\
\hline
\end{tabular}

Table 2: Independent predictors of Intractable Epilepsy after multivariate analysis

\begin{tabular}{|c|c|c|c|c|c|}
\hline S. No & Parameter & Std error & Significance & Odds Ratio & 95\% confidence interval \\
\hline 1 & Sex & 0.416 & 0.017 & 2.68 & $1.18-6.07$ \\
\hline 2 & Multiple seizures before treatment & 0.502 & 0.000 & 9.6 & $3.61-25.87$ \\
\hline 3 & Neurological impairment & 0.698 & 0.0015 & 9.2 & $2.33-36.19$ \\
\hline 4 & Microcephaly & 0.793 & 0.0007 & 14.87 & $3.13-70.50$ \\
\hline 5 & EEG abnormality & 0.439 & 0.000 & 5.52 & $2.33-13.07$ \\
\hline
\end{tabular}

\section{Discussion}

Intractable epilepsy does not have a common agreed upon definition. Definition by Chawla et al is similar to our definition. ${ }^{1}$ The aim of our study was to find out the predictors of intractable epilepsy in childhood so that early identification of these children prone for intractability is possible.

In this study female gender was found to be a risk factor for developing intractable epilepsy in contrast with the results of Javad Akhondian et al, where male gender was found to be a risk factor. ${ }^{4}$ Significant association with $p$ value 0.000 was found between age at onset of seizures less than 1 year and intractability. This is similar to the results of Chawla et al and Javad Akhondian et al. ${ }^{1,4}$

In this study, generalized seizures were more common than partial seizures among cases and controls. Presence of myoclonic epilepsy was associated with intractability with a $\mathrm{p}$ value of 0.006 . This is in concordance with results of Javad Akhondian et al and Chawla et al. ${ }^{4,1}$ However Camfield et al didn't find seizure type as a predictor of intractable epilepsy even in univariate analysis. ${ }^{12}$ Multiple seizure type before starting treatment was found to be a predictor of intractable epilepsy in our study which is similar to the results of Singhvi et al. ${ }^{5}$
In our study, $68 \%$ of patients with intractable epilepsy had developmental delay which was found to be statistically significant. $36 \%$ of cases also had birth asphyxia in newborn period which was also statistically significant with a $p$ value of 0.001 . We noted as did Sillanpaa et al that status epilepticus and intractable seizures were strongly associated, partly because children who had symptomatic epilepsy were more likely to have an episode of status epilepticus. ${ }^{11}$ This association was also found on the results of Berg et al. $^{2}$ The Odds ratio of 23.5 , suggests that the occurrence of status epilepticus may be of some prognostic significance, it may be a marker for an underlying etiology even if none is detected at the time of initial presentation and evaluation.

Neonatal seizures was found to be a predictor of intractable epilepsy with a $\mathrm{p}$ value of 0.005 with Odds ratio of 4.92 and $95 \%$ CI of $1.50-16.15$. This is comparable to the results of Chawla et al and Berg et al. ${ }^{1,2}$ A history of febrile seizures or family history of epilepsy were not found to be of predictive value for intractability.

The most common examination findings in the study population was microcephaly $(34 \%)$, followed by quadriparesis (26\%) and hemiplegia(14\%), unlike other studies were not found to be a predictor of 
intractable epilepsy. Abnormal neurological examination was found to be a predictor of intractable epilepsy, similar to the results of Chawla et al and Berg et al. ${ }^{1,2}$ CT scan or MRI abnormalities unlike other studies were not found to be a predictor of intractable epilepsy. The most common CT abnormality was cerebral atrophy, followed by gliosis. Common MRI findings were cerebral atrophy, neuronal migration disorders, and corpus callosal agenesis. EEG abnormality was found to be a predictor of intractable epilepsy, with a $p$ value of 0.001 . This is comparable to the results of Singhvi et al. ${ }^{5}$

Most common cause of intractable epilepsy detected was cerebral palsy found in 18 patients. Three had neurocutaneous syndromes out of which two had Tuberous Sclerosis and one had Sturge Weber syndrome. Three patients had neurodegenerative disorders, two patients had neurometabolic disorders and four patients had neuronal migration disorders. Two patient had Lennox Gestaut syndrome and two had post meningitic / post encephalitic sequelae. Etiology could not be found in 15 patients.

Factors associated significantly with intractable epilepsy both by univariate and multivariate analysis were female sex, multiple seizures before starting treatment, neurologic impairment, microcephaly and EEG abnormality

\section{Conclusion}

Factors associated significantly with intractable epilepsy both by univariate and multivariate analysis were female sex, multiple seizures before starting treatment, neurologic impairment, microcephaly and EEG abnormality. Early identification of these children prone to develop intractable seizures is critical for parental counselling, selecting patients for more intensive investigations and treatment, such as early consideration for epilepsy surgery

\section{Source of funding}

None.

\section{Conflict of interest}

None.

\section{References}

1. Chawla S, Aneja S, Kashyap R, Mallika V. Etiology and Clinical predictors of intractable epilepsy. Pediatr Neurol 2002;27:186-91.
2. Berg AT, Levy SR, Novotny EJ, Shinnar S. Predictors of intractable epilepsy in childhood: A case-control study. Epilepsia 1996;37:24-30.

3. Altunbasak S, Herguner O, Burgut HR. Risk factors predicting refractoriness in epileptic children with partial seizures. J Child Neurol 2007:22:195-9.

4. Akhondian J, Heydarian F, Jafari SA. Predictive factors of pediatric intractable seizures. Arch Iran Med 2006;9:236-9.

5. Singhvi JP, Sawhney IMS, Lal V, Pathak A, Prabhakar S. Profile of Intractable Epilepsy in a tertiary referral centre. Neurol India 2000;48:351-6.

6. David R Chabolla. Medically refractory seizures. Jacksonville Med 2000;12:45-56.

7. AS Girija. Medical management of intractable epilepsy. Calicut Med J 2004;2(4):e6

8. Kleigman, Behrman, Jenson, Stanton: Nelson textbook of Paediatrics 18: 2457-73

9. Gerald M Fenichel: Clin Paediatr Neurol 5:19-43

10. Al Hail H, Sokrab T, Hamad A.Epidemiology and etiology of intractable epilepsy in Qatar. Qatar Med J 2004:13:1

11. Sillanpaa M. Remission of seizures and prediction of intractability in long term follow up. Epilepsia 1993;34:930-6.

12. Camfield C, Camfield P, Gordon K, Smith B, Dooley J. Outcome of childhood epilepsy: A population based study with a simple scoring system for those treated with medication. J Pediatr 1993;122:861-8

13. Kwan P, Brodie MJ. Early identification of refractory epilepsy. N Engl J Med 2000;342:314e9.

14. Ohtsuka Y, Yoshinaga H, Kobayashi K. Refractory childhood epilepsy and factors related to refractoriness. Epilepsia 2000;41:14e7.

15. Commission on Classification and Terminology of the International League Against Epilepsy. Proposal for revised clinical and electroencephalographic classification of epileptic seizures. Epilepsia 1981;22:489e501.

16. .Engel J Jr, International League Against Epilepsy (ILAE). A proposed diagnostic scheme for people with epileptic seizures and with epilepsy: Report of the ILAE Task Force on Classification and Terminology. Epilepsia 2001;42:796e803.

17. Commission on Classification and Terminology of the International League Against Epilepsy. Proposal for revised classification of epilepsy and epileptic syndromes. Epilepsia 1989;30:389e99.

18. Eriksson KJ, Koivikko MJ. Prevalence, classification and severity of epilepsy and epileptic syndromes in children. Epilepsia 1997;38:1275e82.

19. Maytal J, Shinnar S, Moshe SL, Alvarez LA. Low morbidity and mortality of status epilepticus in children. Pediatr 1989;83:323e31.

20. Gururaj A, Sztriha L, Hertecant J, Eapen V. Clinical predictors of intractable childhood epilepsy. J Psychosom Res 2006;61:343e7.

How to cite: Sangeetha P, Anoop K. Predictors of intractable epilepsy in childhood: A case control study. IP Int J Med Paediatr Oncol 2020;6(1):23-6. 